\title{
WIENER PAIRS OF MEASURE ALGEBRAS
}

\author{
C. KARANIKAS
}

This article concerns the relationship between the spectral and the norm properties of certain subalgebras of the convolution measure algebra $M(G)$ (Borel regular finite measures) on a locally compact amenable group $G$.

1. Introduction. In this paper we deal with the same general problem as in [2] and [4] which is concerned with the relationship of the spectrums of a $C^{*}$-enveloping algebra $C^{*}(A)$ of a subalgebra $A$ of $M(G)$. In different terminology this is the Wiener-Pitt phenomenon for $A$, i.e., whether the spectral radius of $A$ is equal to the norm of the Fourier transform of $A$. Or in Y. Naimark's terminology whether $A$ and $C^{*}(A)$ form a Wiener pair.

In the above mentioned works it has been examined for the case of compact non-Abelian groups $G$. Also the earlier paper [7] deals with the case of Abelian groups.

In the present work we examine this problem for amenable groups $G$ for more general subalgebras of $M(G)$ than those of [2] and [4]. Our methods here differ considerably from previous works. For example one can see that the proof of Theorem (2.6) doesn't make use of the properties of compact groups as Theorem (4.3) of [4].

In $\S 2$ we show that the Fourier norm of the direct sum of group algebras in $M(G)$ coincides with the norm of the left regular representation of $M(G)$ provided that $G$ is an amenable group.

In $\S 3$ we deal with the equality of these norms for more general direct sum of group algebras and we discuss the problem of symmetric subalgebras of $M(G)$. In particular in Theorem (3.3) we give conditions for the symmetry of certain subalgebras of $M(G)$. The conditions involve the equality of the spectral radius with the Fourier norm as well as the equality of spectrums.

We begin in $\S 2$ with a brief presentation of the main definitions and symbols. For the general background, and for definitions and results not given explicit mention here, we refer to [1] and [3]. 
2. On the direct sum of group algebras. Throughout this work $G_{\tau}$ is a locally compact amenable group (see [5]) whose underlying abstract group is $G$ and whose underlying topology is $\mathscr{F}_{\tau}$.

The discrete group of $G$ is denoted by $G_{d}$ and the topological group with the initial (weak) topology is denoted for simplicity by $G$.

We shall denote by $P, P_{d}$ and $P_{\tau}$, the sets of all positive definite functions (p.d. functions) $f$ such that $f(e)=1$ ( $e$ being the unit of $G$ ) and $f$ is continuous on $G, G_{d}$ and $G_{\tau}$ respectively.

Let

$$
\|\mu\|^{\prime}=\sup _{f \in P}\left(\int_{G} f(t) d \mu^{\sim} \cdot \mu(t)\right)^{1 / 2}, \quad \mu \in M(G),
$$

be the norm in $M(G)$ of the left regular representation on $G$, where by - we denote the convolution multiplication and by $\mu \rightarrow \mu^{\sim}$ the usual involution on $M(G)$.

The Fourier norm of a $\sim$ subalgebra $A$ of $M(G)$ will be

$$
\|\mu\|_{A}^{\prime}=\sup \left\langle F, \mu^{\sim} \cdot \mu\right\rangle^{1 / 2}, \quad \mu \in A,
$$

where the supremum is taken over all positive forms $F$ of $A$. For simplicity we denote by $\|\cdot\|_{\tau}^{\prime}$ the norm $\|\cdot\|_{L_{1}\left(G_{\tau}\right)}^{\prime}$.

The next Lemma is the nondiscrete analogue case of Propositions (3.4) and (4.2) in [4].

LEMMA (2.1). If $G_{\tau}$ is an amenable group then for each $\mu \in L_{1}\left(G_{\tau}\right)$, we have:

$$
\|\mu\|_{\tau}^{\prime}=\|\mu\|^{\prime} .
$$

In fact, since $G_{\tau}$ is amenable for any positive $v \in M\left(G_{\tau}\right),\|v\|=\|v\|_{\tau}^{\prime}$ (see [6], Chapter 8, 3.6); thus if $v$ is in $L_{1}\left(G_{d}\right),\|v\|=\|v\|_{d}^{\prime}$ and so $G_{d}$ is amenable too. Since $L_{1}\left(G_{\tau}\right)$ has an approximate identity each element $\mu$ of it can be approximated in the $\|\cdot\|^{\prime}$ and $\|\cdot\|_{\tau}^{\prime}$ norms by elements $v$ of $L_{1}\left(G_{d}\right)$. By Proposition (3.4) of [4], we have $\|v\|_{\tau}^{\prime}=\|v\|^{\prime}$ and so Lemma (2.1) follows.

We can easily see a partial converse of Lemma (2.1) in case where $G$ is a locally compact nondiscrete group $G$ which contains a free group $F$ on two generators. In this case it is obvious that $G$ and $G_{d}$ are nonamenable. In fact if $1_{F}(x)=1$ on $F$ and 0 otherwise the equality of the norms $\|\cdot\|^{\prime}$ and $\|\cdot\|_{d}^{\prime}$ should imply that $1_{F}$ can be approximated on finite sets by continuous p.d. function $\varphi$ with compact support. Let $K \subseteq F$ be finite and $\varepsilon>0$; there exists a $\varphi$ as above such that 
$\left|1_{F}(x)-\varphi(x)\right|<\varepsilon, x \in K$. But this implies that $F$ is amenablecontradiction.

As an example consider $G=\operatorname{SL}(2, R)$ and $F$ any free subgroup of it.

For the rest of this section let $A$ be the algebra $L_{1}\left(G_{\tau}\right) \oplus L_{1}(G)$. It is easy to see that $L_{1}(G)$ is a two sided ideal in $A$. Let $f$ be a positive form on $L_{1}(G)$, i.e., for any $\mu \in L_{1}(G)\left\langle f, \mu^{\sim} \cdot \mu\right\rangle \geq 0$, and $\pi$ be the corresponding representation of $L_{1}(G)$ on a Hilbert space $H$, i.e., $f(\mu)=\langle\pi(\mu) \xi, \xi\rangle, \xi \in H$. It is elementary to see that:

LEMMA (2.2). There exists a representation $\pi^{\prime}$ which extends $\pi$ on $A$, such that $\pi\left(L_{1}(G)\right)$ is strongly dense in $\pi^{\prime}(A)$.

Proof. Given any $\mu \in L_{1}(G)$ such that $f(\mu) \neq 0$ we consider the positive form $f^{\prime}(v)=f\left(\mu^{\sim} \cdot v \cdot \mu\right)$ on $A(v \in A)$. Now let $\pi^{\prime}$ be the representation on a Hilbert space $H^{\prime}$ corresponding to $f^{\prime}$. For any $v \in L_{1}(G)$ :

$$
f^{\prime}(v)=\left\langle\pi^{\prime}(v) \pi^{\prime}(\mu) \xi^{\prime}, \pi^{\prime}(\mu) \xi^{\prime}\right\rangle=\langle\pi(v) \pi(\mu) \xi, \pi(\mu) \xi\rangle .
$$

Thus we may assume that $\pi$ is the restriction of $\pi^{\prime}$ on $H$ and that $f$ can be extended to a positive form $f^{\prime}$ on $A$. The density of $\pi\left(L_{1}(G)\right)$ in $H^{\prime}$ is due to the fact that $L_{1}(G)$ is an ideal with an approximate identity.

Notation. Given a positive form $f$ in $L_{1}(G)$, we shall denote by $f_{\sim}$ a corresponding continuous p.d. function on $G$ and by $f^{\prime}$ the following positive form on $L^{\infty}\left(G_{\tau}\right)$ :

$$
f^{\prime}(\mu)=\int f_{\sim}(t) d \mu(t), \quad \mu \in L_{1}\left(G_{\tau}\right) .
$$

LeMMA (2.3). Let $\left(f_{i}\right)_{i \in I}$ be a net of positive forms on $L_{1}(G)$ which converges weak ${ }^{*}$ in $L^{\infty}\left(G_{\tau}\right)$ to $g^{\prime}$.

Proof. Let $\pi$ be the representation on $L_{1}(G)$, associated with $g$. Since $L_{1}(G)$ is a two sided ideal in $A$, there is a unique extension $\pi^{\prime}$ of $\pi$ on $A$ such that $\pi\left(L_{1}(G)\right)$ is strongly and so weakly dense in $\pi^{\prime}(A)$ (Lemma (2.2)). Thus given $\varepsilon>0$ and $\mu \in L_{1}\left(G_{\tau}\right)$, there is a $v \in L_{1}(G)$ such that

$$
\left|g^{\prime}(\mu)-g(v)\right|<\varepsilon
$$


also for any $i \in I$

$$
\begin{aligned}
\left|f_{i}^{\prime}(\mu)-g^{\prime}(v)\right| \leq & \left|f_{i}^{\prime}(\mu)-f_{i}(v)\right|+\left|f_{i}(v)-g(v)\right| \\
& +\left|g(v)-g^{\prime}(\mu)\right|
\end{aligned}
$$

Since $f_{i}$ converges to $g$, by (1) there is an $i_{0}$ such that for $i \geq i_{0}$,

$$
\left|f_{i}(v)-g(v)\right|+\left|g(v)-g^{\prime}(\mu)\right|<2 \varepsilon .
$$

Hence (2) depends only on the first term of the right hand side. Let now $F_{i}$ be the extension of $f_{i}$ on $A \cdot i \in I$; we consider the cases that either for some $i \geq i_{0},\left|F_{i}(\mu-v)\right|<\varepsilon$ or not. In the first case it follows from (2) and (3) that for some $i \geq i_{0}$

$$
\left|f_{i}^{\prime}(\mu)-g(\mu)\right|<\varepsilon .
$$

In the second case we observe that the set $\left(F_{i}\right)_{i \geq i_{0}}$ is in a compact subset of the dual of $A$ in the weak ${ }^{*}$ topology. Hence there is a subnet of $\left(f_{i}\right)_{i}$, which converges in the weak* topology of $L^{\infty}\left(G_{\tau}\right)$ to $g^{\prime}$. Thus for some $i$, we obtain (4) and this completes the proof.

Lemma (2.4). Let $f \in P$. Then $f$ can be approximated in the uniform topology on compact sets on $G_{\tau}$ by elements of $P_{\tau} \backslash P$.

Proof. Let $K$ be a compact set in $G_{\tau}$; without loss of generality we may assume that $K$ has nonempty interior. We shall show that $f$ coincides in $K$ with an element $g \in P_{\tau} \backslash P$.

First observe that the group $G p K$ generated by $K$ in $G_{\tau}$ cannot be all the group $G$. Because in this case, the topologies of $G$ and $G_{\tau}$ would coincide. We consider the following p.d. function $g(t)$ on $G: g(t)=f(t)$ when $t \in G p K$ and $g(t)=0$ otherwise.

Since $G p K$ is open and $f$ is continuous on $G_{\tau}$, the set

$$
\{t:|g(t)-1|<\varepsilon\}=\{t:|f(t)-1|<\varepsilon\} \cap G p K
$$

is open in $G_{\tau}$. Thus $g$ being continuous at $e$ is continuous in $G_{\tau}$. It is easy to see that $g$ is discontinuous at $G$. Thus $g$ coincides with $F$ on $K$ and $g \in P_{\tau} \backslash P$.

LEMMA (2.5). Let $\left(F_{i}\right)_{i \in I}$ be a net of positive forms on $A$ such that for any $i \in I, F_{i} / L_{1}(G)=f_{i} \neq 0$. Let $F_{i} / L_{1}\left(G_{\tau}\right)=f_{i}^{\prime}, i \in I$, and suppose that $\left(f_{i}^{\prime}\right)$ converges in the weak ${ }^{*}$ topology of $L^{\infty}\left(G_{\tau}\right)$ to some positive form $\mathbf{g}$ such that $g$ is discontinuous in $G$. Then there is a subnet of $\left(f_{i}\right)_{i}$ which converges weak in $L^{\infty}(G)$ to 0 .

Proof. We suppose that there exists a $\mu \in L_{1}(G)$ and a $c>0$ such that for some $i_{0} \in I$, and any $i \geq i_{0},\left|f_{i}(\mu)\right| \geq c$. It is clear that the 
set $\left(f_{i}\right)_{i \geq i_{0}}$ is in a weak* compact subset of $L^{\infty}(G)$. Thus this net has a convergent subnet with limit (say) $h$. By Lemma (2.5) there exists a subnet of $\left(f_{i}^{\prime}\right)_{i}$ which converges weak ${ }^{*}$ in $L^{\infty}\left(G_{\tau}\right)$ to $h^{\prime}$. Clearly $g=h$ and so $\mathbf{g}$ is $G_{\tau}$-continuous-contradiction. Thus for any $c>0$ and any $i_{0} \in I$ there is an $i \geq i_{0}$, such that $\left|f_{i}(\mu)\right|<c$ and so there is a subnet as we claimed.

Theorem (2.6). For any $\mu \in A$ (provided that $G_{\tau}$ is amenable)

$$
\|\mu\|_{A}^{\prime}=\|\mu\|^{\prime} .
$$

Proof. Let $\mu=\mu_{\tau}+\mu_{\alpha}$ where $\mu_{\tau} \in L_{1}\left(G_{\tau}\right)$ and $\mu_{\alpha} \in L_{1}(G)$. Let also $\varepsilon>0$ is given. There exists a positive form $F$ on $A$ such that

$$
\left(\|\mu\|^{\prime}\right)^{2}<\left\langle F, \mu^{\sim} * \mu\right\rangle+\varepsilon .
$$

Let $f$ an $f^{\prime}$ be the p.d. functions corresponding to $F / L_{1}(G)$ and $F / L_{1}\left(G_{\tau}\right)$ respectively. We consider the following cases:

(a) $f \neq 0$ and so $f$ is continuous in $G$.

(b) $f=0$.

If $F$ is as in case (b), then by Lemma (2.1) and Lemma (2.3), there is a positive form $H, H / L_{1}(G)=0$ such that if $h$ is the corresponding continuous p.d. function on $G_{\tau}$, we have

$$
\left|\int f^{\prime}(t) d \mu_{\tau}^{\sim} * \mu_{\tau}(t)-\int h(t) d \mu_{\tau}^{\sim} \cdot \mu_{\tau}\right|<\varepsilon .
$$

From (1) and (2) it is clear that

$$
\left(\|\mu\|_{A}^{\prime}\right)^{2} \leq\left\langle H, \mu^{\sim} \cdot \mu\right\rangle+\varepsilon \leq\left(\|\mu\|^{\prime}\right)^{2}+2 \varepsilon,
$$

and so since $\varepsilon$ is arbitrarily small

$$
\|\mu\|_{A}^{\prime} \leq\|\mu\|^{\prime} .
$$

If $F$ is as in case (a) then from (1) we obtain (4).

Since $\|\cdot\| \leq\|\cdot\|_{A}^{\prime}$, the norms are as we claimed.

3. Symmetric subalgebras. In this section we consider a more general direct sum of group subalgebras of $M(G)$. We shall establish, as in $\S 2$ the equality of Fourier norms and discuss the problem of the symmetry.

Let $G$ be a nondiscrete locally compact group and $\left(\mathscr{F}_{\tau}\right)_{\tau \in V}$ be a collection of distinct locally compact topologies on the group $G$ such that $V$ is a semi-lattice (inclusion ordered). For any $\tau, \sigma \in V$ there exists $\tau \cap \sigma \in V$ such that $\tau \cap \sigma \leq \tau$ and $\tau \cap \sigma \leq \sigma$ and $\mathscr{F}_{\tau \cap \sigma}=\mathscr{F}_{\tau} \cap \mathscr{F}_{\sigma}$. 
Assume that $o \in V$ is the minimum of $V$, i.e., $G_{o}$ is the initial topology. Assume also that $d \in V$ corresponds to the discrete topology. For any $\tau \in V$, we denote by $G_{\tau}$ the group $G$ with the topology $\mathscr{F}_{\tau}$. For simplicity we write $G$ instead of $G_{o}$.

As in [7] we observe that the algebra $L_{V}=\bigoplus_{\tau \in V} L_{1}\left(G_{\tau}\right)$ is a closed subalgebra of $M(G)$. It is clear that $L_{1}(G)$ is a two sided ideal in $L_{V}$ and so any positive form on $L_{1}(G)$ has a unique extension on $L_{V}$. We shall show now that the Fourier norm $\|\cdot\|_{V}^{\prime}$ on $L_{V}$, coincides with the $\|\cdot\|^{\prime}$ norm.

Lemma 3.1. Let $\tau, \kappa_{1}, \ldots, \kappa_{p} \in V$ such that $\tau \not \subset q, q=\kappa_{1}, \ldots, \kappa_{p}$. If $h$ is a positive form on $L_{1}\left(G_{\tau}\right)$, then there is a net $\left(F_{i}\right)_{i \in I}$ of positive forms of norm one on $L_{V}: F_{i} / L_{1}(G) \neq 0$, and $F_{i} \rightarrow 0, i \in I$, weak ${ }^{*}$ in $L^{\infty}\left(G_{q}\right), q=\kappa_{1}, \ldots, \kappa_{p}$ and $F_{i} \rightarrow h, i \in I$, weak ${ }^{*}$ in $L^{\infty}\left(G_{\tau}\right)$.

Proof. Let $m=\min \left\{\tau, \kappa_{1}, \ldots, \kappa_{p}\right\}$. Then since $P$ is dense in $P_{\tau}$ by Lemma (2.5), there is a net of positive forms $\left(F_{i}\right)_{i \in I}$ such that $F_{i} \rightarrow h$, $i \in I$ weak ${ }^{*}$ in $L^{\infty}\left(G_{m}\right)$.

We suppose that there is no subnet of it satisfying the Lemma and so we assume that for some $q=\kappa_{1}, \ldots, \kappa_{p}, c \geq 0$ and $\mu \in L_{1}\left(G_{q}\right)$, $\left|F_{i}(\mu)\right| \geq 2 c, i \in I$.

This set is weak ${ }^{*}$ compact and so it has a subnet converging to some positive form $F$. It is clear that $|F(\mu)| \geq 2 c$ and we may write:

$$
\left|F_{i}(\mu)-F(\mu)\right|<c / 3, \quad i \geq i_{0}, i_{0} \in I .
$$

If $q<\tau$, (1) contradicts with Lemma (2.5), so without loss of generality we may assume that $m=\min (q, \tau)$.

Let $\left(v_{j}\right)_{j \in J}$ be an approximate identity in $L_{1}\left(G_{m}\right)$. On $L_{1}\left(G_{q}\right) \oplus$ $L_{1}\left(G_{m}\right)$ the Fourier norm coincides with the $\|\cdot\|^{\prime}$ norm and since $\left\|v_{j} \cdot \mu^{\sim} \cdot \mu\right\|^{\prime} \rightarrow 0, j \in J$, we obtain $F_{i}\left(\left(v_{j} \cdot \mu^{\sim} \cdot \mu\right)^{\sim} \cdot\left(v_{j} \cdot \mu^{\sim} \cdot \mu\right)\right) \rightarrow 0$, $j \in J$, for each $i \in I$. Hence exists some $j_{0} \in J$ such that for each $i \in I$

$$
\left|F_{i}\left(v_{j} \cdot \mu\right)-F_{i}(\mu)\right|<c / 3, \quad j \geq j_{0} .
$$

Now, $v_{j} \cdot \mu \in L_{1}\left(G_{m}\right)$ and $F_{i}\left(v_{j} \cdot \mu\right) \rightarrow 0, i \in I$; thus for some $i \geq i_{0}$, $i_{0}^{\prime} \in I$.

$$
\left|F_{i}\left(v_{j} \cdot \mu\right)\right|<c / 3 .
$$

From (1), (2) and (3) we obtain $|F(\mu)|<c$-contradiction.

Proposition (3.2). For any $\mu \in L_{V}$ (provided that $G_{d}$ is amenable)

$$
\|\mu\|_{V}^{\prime}=\|\mu\|^{\prime} \text {. }
$$


Proof. It suffices to show that any positive form $H$ on $L_{V}$ is the weak* limit of positive forms $F$ such that $F / L_{1}(G) \neq 0$ provided that the norms of $H$ and $F$ 's are one.

Since finite sums $\sum_{\tau \in V} \mu_{\tau}$, where $\mu_{\tau} \in L_{1}\left(G_{\tau}\right)$, are dense in $L_{V}$ we show that given $\varepsilon>0, \mu_{k_{i}} \in L_{1}\left(G_{k_{l}}\right)(i=1, \ldots, n)$ there exists a positive form $F$, as above, such that

$$
\left|H\left(\mu_{k_{l}}\right)-F\left(\mu_{k_{l}}\right)\right|<\varepsilon \quad(i=1, \ldots, n) .
$$

Let $m$ be the minimum index in $V$ such that $H / L_{1}\left(G_{m}\right) \neq 0$. We consider the following cases:

1. $m \leq \min \left(\kappa_{1}, \ldots, \kappa_{n}\right)$. We apply Lemmas $(2.1)$ and (2.5).

2. $m \leq \min \left(\kappa_{1}, \ldots, \kappa_{s}\right)$ and $q \not \leq \kappa_{s+1}, \ldots, \kappa_{n}(1<s \leq n)$. In this case we apply Lemma (4.1) for the set of indexes $m, \kappa_{s+1}, \ldots, \kappa_{n}$, and we continue as in Case 1. Thus the weak ${ }^{*}$ limit of $F$ 's to $H$ implies that $\|\cdot\|_{V}^{\prime}=\|\cdot\|^{\prime}$ on $L_{V}$.

We recall that a Banach algebra $A$ with an involution $\mu \rightarrow \mu^{\sim}$ is symmetric if $\operatorname{sp}\left(\mu^{\sim} \cdot \mu\right) \subseteq \mathbf{R}^{+}$, for any $\mu \in A$. In [4] we have seen the following result on general Banach algebras. If $A$ is a Banach-algebra with identity, and $\varphi: A \rightarrow C^{*}(A)$ is the natural homomorphism of $A$ in the enveloping $C^{*}$ algebra of $A$, and $\rho(\cdot)$ is the spectral radius, then if $\mu \in A$ the next conditions are equivalent:

(i) $A$ is symmetric,

(ii) $\operatorname{sp} \mu=\operatorname{sp}(\varphi(\mu))$,

(iii) $\rho(\mu)=\rho(\varphi(\mu))$,

(iv) $\rho\left(\mu^{\sim} \cdot \mu\right)=\|\varphi(\mu)\|^{2}$.

Now let $A$ be the algebra $L_{V}$. Then $\|\varphi(\mu)\|=\|\mu\|_{V}^{\prime}, \mu \in L_{V}$, and so by Proposition (3.2) we establish a Theorem:

Theorem (3.3). Provided that $G_{d}$ is amenable and $\mu \in L_{V}$, we have the following equivalent conditions:

(i) $L_{V}$ is symmetric,

(ii) $\operatorname{sp} \mu=\operatorname{sp} \varphi(\mu)$,

(iii) $\rho(\mu)=\|\mu\|^{\prime}$,

(iv) $\rho\left(\mu^{\sim} \cdot \mu\right)^{1 / 2}=\|\mu\|^{\prime}$.

For the relation between amenable and Hermitian groups $G$ we refer to [5] ( $G$ is hermitian iff $L_{1}(G)$ is a symmetric algebra).

I would like to express my thanks to the referee for his suggestions. 


\section{REFERENCES}

[1] J. Dixmier, $C^{*}$ Algebras, (North-Holland, 1977).

[2] J. B. Fountain, R. W. Ramsay and J. H. Williamson, Functions of measures on compact groups, Proc. Roy. Irish Acad. Sect. A, 76 (1976), 235-251.

[3] E. Hewitt and K. Ross, Abstract Harmonic Analysis, vols. I, II (Springer-Verlag, 1963, 1970).

[4] C. Karanikas and J. H. Williamson, Norms and spectra for certain subalgebras of $M(G)$, Math. Proc. Camb. Phil. Soc., 95 (1984), 109-122.

[5] T. W. Palmer, Classes of nonabelian, noncompact, locally compact groups, Rocky Mountain J. Math., 8 (1978), 683-741.

[6] H. Reiter, Classical Harmonic Analysis and Locally Compact Groups, Oxford Univ. Press 1968.

[7] N. Varopoulos, Studies in harmonic analysis, Proc. Camb. Phil. Soc., 60 (1964), 465-516.

Received October 23, 1987 and in revised form March 8, 1988.

UNIVERSITY OF THESSALONIKI

ThesSALONIKI, GreECE 